\title{
Nano-glass Mechanism of Bulk Metallic Glass Formation
}

\author{
Takeshi Egami \\ Department of Materials Science and Engineering, University of Pennsylvania, Philadelphia, PA 19104, USA
}

A theory of local dynamics of liquid is developed in order to explain the glass transition and fragility of multi-component alloys as it relates to formation of bulk metallic glasses. Unlike the extended hydrodynamic theories in which liquid is regarded as a continuum body, the present approach focuses on the discreteness of the atomic structure and considers the stability of local topology of the network structure. This approach has led to the prediction of the glass transition temperature, melting and glass formability. We extend this approach to describe the effects of local topology on the atomic transport and glass transition and fragility of multi-component glasses. This theory leads to a picture of a strong liquid as a nano-scale composite of glass and liquid, and suggests compositional requirements for forming bulk metallic glasses.

(Received October 25, 2001; Accepted January 10, 2002)

Keywords: bulk metallic glasses, glass formation, fragility of liquid, glass transition

\section{Introduction}

Liquids and glasses are complex systems and do not allow simple and precise descriptions of their structure. For this reason the progress in understanding the dynamics of liquid and glasses has been sluggish. Only recently notable progress has been achieved in describing the dynamics of liquid by hydrodynamic theories, such as the mode-coupling theory, ${ }^{1,2}$ ) in which liquid is regarded as a continuum body with nonlinear responses, totally disregarding the atomic structure. At high temperatures atomic mobility is high enough to justify such an approximation, and this has been the reason of successes by the hydrodynamic theories. However, as temperature is lowered atomic processes start to dominate the dynamics and transport, and the continuum approximation breaks down. This effect actually was first recognized long time ago by Cohen and Turnbull in their seminal free-volume theory as backscattering of atoms in the cage of nearest neighbors. ${ }^{3-5)}$ The free-volume theory has been very successful in describing the diffusion and flow of glasses, and has been widely used in explaining various properties of glasses and glass transition. ${ }^{6,7)}$

However, both the mode-coupling theory and the freevolume theory are basically phenomenological theories, requiring fitting parameters to be determined by comparison with experiment. We proposed some time ago a more microscopic approach to the question of glass formability, based upon the model of atoms interacting with a pair-wise potential. ${ }^{8)}$ In this approach this problem is addressed from the low temperature side, starting with a discrete network structure of a glass, and introducing a topological criterion of local structural instability. This approach was more recently extended to explain a wide variety of properties, form melting of crystals, glass transition as well as glass formation. ${ }^{9)}$ In this paper we first review the theory of local topological instability, and discuss how this model can be further extended to account for the atomic mobility in multi-component glasses. We suggest that the atomic size effect can results in differential atomic transport properties among the constituent elements, and directly influence the glass transition and fragility of the glass as defined by Angel. ${ }^{10)}$
This work was motivated by the recent development of bulk metallic glasses ${ }^{11-13)}$ and the need of a theory that could guide the effort to develop new bulk metallic glasses. The main purpose of this work is to provide a theoretical basis of alloy selection for bulk metallic glass formation through the microscopic theory of glass transition and viscosity of liquid, based principally upon the atomic size-effect. For this reason we limit our scope to metallic systems that can be described by simple spherical pair-wise potentials, while the theory could be extended to covalent glasses. The fragility of glass or liquid is defined by the dependence of the viscosity on the renormalized temperature, $T / T_{\mathrm{g}}$, where $T_{\mathrm{g}}$ is the glass transition temperature. ${ }^{10)}$ Above $T_{\mathrm{g}}$ a strong liquid has much higher viscosity than the fragile liquid, by many orders of magnitude. This high viscosity in the liquid state retards crystal nucleation and growth, so that being a strong glass is an important requirement for the formation of bulk metallic glasses. ${ }^{14)}$ At this moment it is not clear what factors determine the fragility of a glass, particularly for metallic systems. Our theory qualitatively explains what atomistic conditions lead to a strong glass, and suggests ways of improving the ease of metallic glass formation.

\section{Atomic Transport and Local Topological Instability}

\subsection{Topology of the glass structure}

While the mechanism of atomic diffusion in crystals is well understood, that in liquids and glasses is poorly understood. This is not surprising given the fact that for glasses and liquids it is not easy even to define the atomic structure. Thus usually the diffusion in glasses and liquids is discussed in terms of concepts and terminologies simply borrowed from theories of diffusion in crystals. But this might have produced unjustifiable biases in our thinking. For instance, in crystals diffusion occurs by vacancy and interstitial defect mechanisms. Thus various attempts have been made to define defects in a similar way. The most well-known definition of "vacancy" in glasses and liquids is the free-volume. ${ }^{3-5)}$ However, in glasses defects cannot be uniquely defined, since there is no reference structure to define defects against. This is a part of the reasons why the free-volume theory has remained a phenomenologi- 
cal theory. A useful and unambiguous definition of structural defects has eluded us so far.

Another bias in discussing diffusion is to focus on the motion of a single atom. This may appear as a natural bias that could make the argument simple. But in fact this view is closely tied to the periodic nature of the lattice, and in the absence of a periodic lattice its validity is not obvious. To prove this point let us consider a simple crystal with the Bravais lattice. In such crystals the structures before and after a single diffusion jump have to be the same, except that the position of the defect is displaced by the lattice periodicity, if we neglect the presence of other defects and boundaries. Therefore the magnitude of the atomic jump has to be of the order of the interatomic distance. In glasses or liquids, the structures before and after the atomic motion are not the same in the absence of lattice periodicity, and therefore there is no limit in the magnitude of atomic displacement. Actually it is more likely that the motion at a time is a fraction of the atomic distance, since atomic motions are likely to be strongly correlated. If one atom moves in one direction, the vacated space is likely to be filled by another atom moving in the opposite direction, resulting in local ring-like atomic motion. In crystals such a ring-like motion does not produce a net atomic transport. In glasses and liquids, however, the displacements of these two atoms will not exactly cancel each other, resulting in fractional atomic transport. These examples illustrate the need of going sufficiently away from the picture of single-atom diffusion in the crystalline structure to describe diffusion in liquids and glasses. Different mechanisms that are uniquely appropriate for liquids and glasses might exist.

In order to find diffusion processes unique to glasses and liquids, let us discuss how to define the structure of glasses in a way meaningful in discussing their atomic transport. In the present work we define the structure topologically as a network in terms of atomic connectivity. Such a definition is a standard method to describe covalently bonded glasses, ${ }^{15)}$ but it can be extended to metallic glasses as well, since the negative curvature of the interatomic potential tends to separate the nearest and second nearest neighbors clearly, as argued by Turnbull and Cohen in their discussion on the free-volume theory. ${ }^{5)}$ Let us consider an atom interacting via a two-body potential $V(r)$ with a minimum at $r=2 a$. If $r$ is larger than the position of the inflection point, $r_{0}\left(d^{2} V\left(r_{0}\right) / d r^{2}=0\right)$, this atom will not stay at the mid-point of the two neighbors. This is because the system lowers its energy when this atom moves away from the center. This illustrates how the interatomic potential naturally leads to better definition of the nearest neighbors.

Once the structure is defined as a topological network any structural change, including diffusion, can be defined in terms of the changes in connectivity (bonds) of atoms. This is an important point of departure from focusing on the motion of a single atom. Usually when a bond is broken-off, a new bond is formed elsewhere without changing the total density of bonds. Thus changes in structure or atomic transport can be defined in terms of the bond exchange. For instance the plastic shear process and anelastic strain can be described in such terms. ${ }^{16,17)}$

\subsection{Instability of local topology}

The next question is at what condition the atomic bond will be broken. To answer this question it is instructive to consider the equilibrium local coordination number of an impurity atom $A$ in the host $B$ liquid or glass, as a function of the atomic radius of $A, r_{A}$, and that of $B, r_{B}$. In liquids and glasses the local coordination number depends upon the atomic size ratio, $x=r_{A} / r_{B}$, since it is not a fixed number. When $x$ is small, a relatively small number of host atoms can be the nearest neighbors of the impurity atom, while when $x$ is large the number of the neighbors should be large as well. When viewed from the $A$ atom as shown in Fig. 1, the solid angle occupied by a neighboring $B$ atoms which is in contact with the $A$ atom is,

$$
\begin{aligned}
\omega_{A-B}(x) & =2 \pi\left[1-\frac{\sqrt{r_{A}\left(r_{A}+2 r_{B}\right)}}{r_{A}+r_{B}}\right] \\
& =2 \pi\left[1-\frac{\sqrt{x(x+2)}}{x+1}\right] .
\end{aligned}
$$

Since atoms are spherical, they cannot fill the space even at the most efficient packing. The efficiency of packing $B$ atoms on the surface of the $A$ atom can be expressed by the 2-dimensional packing density,

$$
\eta_{2 D}=\frac{N_{\mathrm{C}}^{A}(x) \omega_{A-B}(x)}{4 \pi},
$$

where $N_{\mathrm{C}}^{A}(x)$ is the average coordination number of an $A$ atom. It is reasonable to assume that $\eta_{2 D}$ is independent of $x$, if $x$ is not too far from unity. Indeed this was validated by simulation with a simple alloy model. ${ }^{18)}$ The simulation ${ }^{18)}$ furthermore has shown that,

$$
\eta_{2 D}=2 \pi\left[1-\frac{\sqrt{3}}{2}\right]=\omega_{A-B}(1) .
$$

Therefore, from (1)-(3) we obtain,

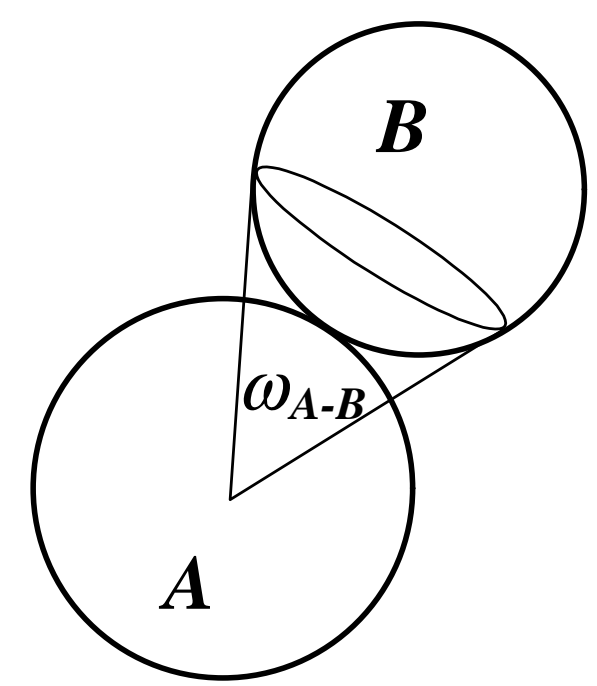

Fig. 1 Solid angle $\omega_{A-B}$ to view a $B$ atom from an $A$ atom. 


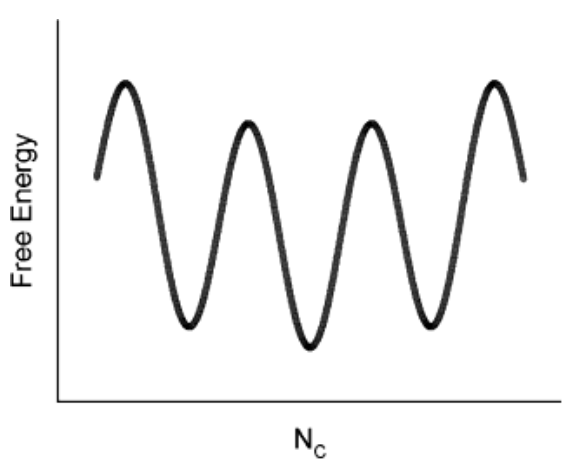

Fig. 2 Energy landscape for the change in the local coordination.

$$
\begin{aligned}
N_{\mathrm{C}}^{A}(x) & =4 \pi\left[1-\frac{\sqrt{3}}{2}\right] /\left[1-\frac{\sqrt{x(x+2)}}{x+1}\right] \\
& =2 \eta_{2 D} /\left[1-\frac{\sqrt{x(x+2)}}{x+1}\right] .
\end{aligned}
$$

Note that for a single component system $(x=1)$ this gives $N_{\mathrm{C}}^{A}(1)=4 \pi$, and the average solid angle per neighbor to be unity.

Now let us consider a gedanken experiment in which we start from the equilibrium state, and increase the radius of the $A$ atom continuously. The question is how long the environment can remain topologically unchanged? Since $N_{\mathrm{C}}$ is discreet a small change in the value of $x$ by $\Delta x$ will simply result in the elastic expansion of the environment, and will not cause a topological change. However, if $x+\Delta x$ is sufficiently large so that the corresponding value of $N_{\mathrm{C}}$ is as large as $N_{\mathrm{C}}+1$, the environment will collapse through bond exchange, and the coordination number will be increased by one. The critical value of $\Delta x$ is given by

$$
\frac{\partial N_{\mathrm{C}}^{A}(x)}{\partial x} \Delta x=1 \text {. }
$$

At high enough temperatures, however, one does not have to increase $x$ by this amount, and only one half of this will do. The reason is that the free energy as a function of $x$ is a corrugated function, as illustrated in Fig. 2. As $\Delta x$ is increased the value of $x+\Delta x$ goes over the potential barrier, and the system will undergo a first-order transition and settle to the next value of $N_{\mathrm{C}}$. Thus the critical value of $\Delta x, \Delta x_{\mathrm{C}}$, is,

$$
\Delta x_{\mathrm{C}}=\frac{1}{2} / \frac{\partial N_{\mathrm{C}}^{A}(x)}{\partial x} \text {. }
$$

For a single component system $(x=1)$,

$$
\left.\frac{\partial N_{\mathrm{C}}^{A}(x)}{\partial x}\right|_{x=1}=\frac{2 \pi^{2} \sqrt{3}}{3 \eta_{2 D}} .
$$

Thus the topological instability condition is expressed as,

$$
\Delta x_{\mathrm{C}}=\frac{\sqrt{3}}{4 \pi^{2}} \eta_{2 D}
$$

We now apply this result to explain the development of local structural instability due to thermal volume expansion $\varepsilon_{\mathrm{V}}$. In a single component system $\left(r_{A}=r_{B}\right)$ the nearest neighbor atomic distance $2 r_{A}$ becomes $2 r_{A}\left(1+\varepsilon_{\mathrm{V}} / 3\right)$ because of thermal expansion. If we assign all the expansion to the change in the radius of the central $A$ atom, or the change in $x$, the
$A-B$ distance becomes $r_{A}+r_{B}=r_{B}(1+\Delta x)+r_{B}=$ $2 r_{B}(1+\Delta x / 2)$. Therefore $\varepsilon_{\mathrm{V}} / 3=\Delta x / 2$, or $\varepsilon_{\mathrm{V}}=3 \Delta x / 2$. Thus the critical volume expansion for local topological stability is,

$$
\varepsilon_{\mathrm{V}}^{\text {crit }}=\frac{3}{2} \Delta x_{\mathrm{C}}=\frac{3 \sqrt{3}}{8 \pi^{2}} \eta_{2 D}=\frac{6 \sqrt{3}-9}{8 \pi}=0.0554 .
$$

Indeed most of elemental metals melt near this volume expansion. ${ }^{9,19)}$ The criterion (9) is related to the well-known Lindemann's criterion ${ }^{20)}$ through anharmonicity. This result was the basis to calculate the minimum solute composition to form a metallic glass of a binary system, $\left.c_{A}^{\min } \cdot{ }^{8}\right)$

\subsection{Local structural quantization and glass transition}

The local topological instability caused by local expansion beyond the critical value $\varepsilon_{\mathrm{V}}^{\text {crit }}$ results in collapse of the environment and the change in the local coordination. A liquid can be defined as a state in which such a local catastrophe is taking place all the time everywhere, with the time-scale of the inverse of the Debye frequency. Thus the time-averaged value of $N_{\mathrm{C}}$ is a non-integral number. As the temperature is lowered the volume fluctuation decreases, and the local coordination falls into one of the valleys of the free-energy landscape shown in Fig. 2, and the value of $N_{\mathrm{C}}$ becomes locally locked into an integral number for a while. In other words as temperature is lowered $N_{\mathrm{C}}$ becomes locally "quantized" into integral values. This crossover from the fluctuating, time-dependent local coordination to the discrete local coordination characterizes glass transition.

The condition for the crossover can be determined from the local dynamics of the liquid state. Firstly we propose to describe the local structural fluctuation in liquids and glasses in terms of the local atomic-level stresses. ${ }^{21)}$ This concept originated from the realization that in liquids and glasses most of the interatomic distances are non-ideal, being either stretched or compressed, resulting in the two-body forces between the atoms. The local sum of the two-body forces,

$$
f^{\alpha}(i)=\frac{1}{\Omega_{i}} \sum_{j} f_{i j}^{\alpha},
$$

where $f_{i j}^{\alpha}$ is the $\alpha$ Cartesian component of the force between the atoms $i$ and $j$ and $\Omega_{i}$ is the local atomic volume of atom $i$, is zero in an equilibrium structure, but the local atomic-level stress tensor,

$$
\sigma^{\alpha, \beta}(i)=\frac{1}{\Omega_{i}} \sum_{j} f_{i j}^{\alpha} r_{i j}^{\beta},
$$

where $r_{i j}^{\alpha}$ is the $\alpha$-component of the separation vector $\boldsymbol{r}_{i j}$ between atoms $i$ and $j$, is not zero except in the Bravais lattice at $T=0$. The stress tensor $\overline{\bar{\sigma}}$ has six independent components that can be grouped into one pressure $\left(p_{i}, l=0\right)$ and five shear $\left(\tau_{i}, l=2\right)$ components. Such a local stress produces a local strain tensor,

$$
\begin{aligned}
p_{i} & =\operatorname{Tr}(\overline{\bar{\sigma}}), \quad \tau_{i}^{m}=\frac{1}{\Omega_{i}} \sum_{j} Y_{2}^{m}\left(\frac{\boldsymbol{r}_{i j}}{r_{i j}}\right) f_{i j} r_{i j}, \\
\tau_{i} & =\left(\frac{1}{5} \sum_{m}\left(\tau_{i}^{m}\right)^{2}\right)^{1 / 2},
\end{aligned}
$$




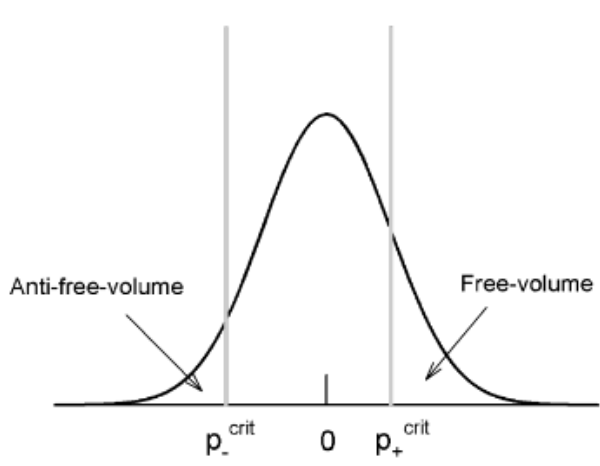

Local Pressure

Fig. 3 Distribution of the atomic level strain and the definition of the free-volume and anti-free-volume.

where $f_{i j}=\left|\boldsymbol{f}_{i j}\right|, r_{i j}=\left|\boldsymbol{r}_{i j}\right|, Y_{2}^{m}$ is the spherical harmonics, and the associated elastic energy,

$$
E=\frac{\Omega}{2 K} \sum_{i} p_{i}^{2}+\frac{5 \Omega}{4 G} \sum_{i} \tau_{i}^{2},
$$

where $K$ and $G$ are bulk and shear moduli. Here the fluctuations in the local volume and local moduli have been neglected. Due to the virial theorem a half of the thermal energy, $3 k T$, goes to the potential energy, while the rest becomes the kinetic energy. In the liquid state the potential energy, $(3 / 2) k T$, is equally divided into six independent components of the atomic-level stress energies. ${ }^{22,23)}$ Thus the average energy of volume fluctuation is given by

$$
\frac{\Omega}{2 K}\left\langle p^{2}\right\rangle=\frac{\Omega}{4 G}\left\langle\tau^{2}\right\rangle=\frac{k T}{4} .
$$

Accordingly the local pressure, thus the local volume strain, exhibits a gaussian distribution with the standard deviation,

$$
\begin{gathered}
\Delta p=\left\langle p^{2}\right\rangle^{1 / 2}=\left(\frac{K k T}{2 \Omega}\right)^{1 / 2}, \\
\Delta \varepsilon_{\mathrm{V}}=\left\langle\varepsilon_{\mathrm{V}}^{2}\right\rangle^{1 / 2}=\left(\frac{k T}{2 K \Omega}\right)^{1 / 2} .
\end{gathered}
$$

Note that eqs. (13) and (14) are valid only above $T_{\mathrm{g}}$, while in the glassy state local stresses and strains remain higher than these since the system becomes non-ergodic.

Now let us consider the dynamics of a liquid of which local volume strain has the gaussian distribution according to (14) (Fig. 3). If the local volume strain is smaller than the critical value, $\varepsilon_{\mathrm{V}}^{\text {crit }}$, then such an atom is "solid-like", and its coordination has a long life-time. On the other hand if it is larger, the atom is "liquid-like", and its coordination is unstable and changes within the Debye time. Thus the system is divided into liquid-like and solid-like regions, as envisaged by Cohen and Grest. ${ }^{24,25)}$ Actually this is the re-definition of the free-volume in the present language. Now, as shown in Fig. 3 the critical strain can be exceeded on both the positive side (local free-volume) and the negative side (local anti-freevolume). ${ }^{26)}$ However, the anharmonicity in the potential and the asymmetry in eq. (1) with respect to deviations make the anti-free-volume much less effective. It can be readily shown, for instance, that for a reasonable value of the Grüneisen constant of 2, the amount of anti-free-volume is only $1 / 3$ of the free-volume. Here for simplicity we will neglect the effects of anti-free-volume on the dynamics of liquids.

The volume fraction of the atoms with the local volume larger than the critical value, $\xi$, is given by,

$$
\xi=\frac{1}{\sqrt{2 \pi} \Delta \varepsilon_{\mathrm{V}}} \int_{\Delta \varepsilon_{\mathrm{V}}^{\text {crit }}}^{\infty} \exp \left(-\frac{\varepsilon^{2}}{2 \Delta \varepsilon_{\mathrm{V}}^{2}}\right) d \varepsilon
$$

Glass transition can now be seen in terms of percolation of the liquid-like regions. Assuming the percolation concentration in three-dimensional dense-packed systems to be about $0.15,{ }^{27)}$ we obtain the percolation condition to be,

$$
T_{\mathrm{g}}=\frac{2 \Omega K}{k_{\mathrm{B}}}\left(\varepsilon_{\mathrm{V}}^{\text {crit }}\right)^{2}=6.14 \times 10^{-3} \frac{\Omega K}{k_{\mathrm{B}}}
$$

which applies well for a large number of metallic glasses. ${ }^{28)}$

\section{Local Dynamics of Multi-Component Glasses}

\subsection{Local structure of multi-component glasses}

In the argument above a single component glass was assumed. We now discuss the case of multi-component glasses. Let us consider a binary glass of transition metal $(T)$ and metalloid $(M), T_{1-y} M_{y}$, with the size ratio, $z=r_{M} / r_{T}<1$. A well known example is $\mathrm{Fe}_{0.8} \mathrm{~B}_{0.2}$. Since a $T$ atom is larger than an $M$ atom, its coordination number, $N_{\mathrm{C}}^{T}$, will be larger than that of $M, N_{\mathrm{C}}^{M}$. We also have to differentiate the neighbors according to chemistry. So the average number of $T$ atom neighbors that are in contact with the $M$ atom at the center is denoted as $N_{\mathrm{C}}^{M-T}$, etc. Thus,

$$
\begin{aligned}
& N_{\mathrm{C}}^{T}=N_{\mathrm{C}}^{T-T}+N_{\mathrm{C}}^{T-M}, \\
& N_{\mathrm{C}}^{M}=N_{\mathrm{C}}^{M-T}+N_{\mathrm{C}}^{M-M}
\end{aligned}
$$

Note that,

$$
y N_{\mathrm{C}}^{M-T}=(1-y) N_{\mathrm{C}}^{T-M} .
$$

In the absence of compositional short-range order, ${ }^{29)}$

$N_{\mathrm{C}}^{i j}=c_{j} N_{\mathrm{C}}^{i} N_{\mathrm{C}}^{j} /\left\langle N_{\mathrm{C}}\right\rangle, \quad\left\langle N_{\mathrm{C}}\right\rangle=y N_{\mathrm{C}}^{M}+(1-y) N_{\mathrm{C}}^{T}$,

where $c_{M}=y$ and $c_{T}=1-y$. The eq. (2) is now,

$$
\begin{aligned}
\eta_{2 D} & =\frac{1}{4 \pi}\left[N_{\mathrm{C}}^{T-T} \omega_{T-T}+N_{\mathrm{C}}^{T-M} \omega_{T-M}\right] \\
& =\frac{1}{4 \pi}\left[N_{\mathrm{C}}^{M-T} \omega_{M-T}+N_{\mathrm{C}}^{M-M} \omega_{M-M}\right],
\end{aligned}
$$

where,

$$
\begin{aligned}
& \omega_{T-T}=\omega_{M-M}=2 \pi\left[1-\frac{\sqrt{3}}{2}\right]=\eta_{2 D}, \\
& \omega_{M-T}=2 \pi\left[1-\frac{\sqrt{z(z+2)}}{1+z}\right], \\
& \omega_{T-M}=2 \pi\left[1-\frac{\sqrt{1+2 z}}{1+z}\right] .
\end{aligned}
$$

\subsection{Local feezing}

We now consider how much local volume expansion is needed to change the local coordination. Taking the variation of (21), 


$$
\begin{aligned}
& \delta \eta_{2 D}=\frac{1}{4 \pi}\left[\delta N_{\mathrm{C}}^{T-T} \omega_{T-T}+N_{\mathrm{C}}^{T-T} \delta \omega_{T-T}+\delta N_{\mathrm{C}}^{T-M} \omega_{T-M}+N_{\mathrm{C}}^{T-M} \delta \omega_{T-M}\right]=0, \\
& \delta \eta_{2 D}=\frac{1}{4 \pi}\left[\delta N_{\mathrm{C}}^{M-T} \omega_{M-T}+N_{\mathrm{C}}^{M-T} \delta \omega_{M-T}+\delta N_{\mathrm{C}}^{M-M} \omega_{M-M}+N_{\mathrm{C}}^{M-M} \delta \omega_{M-M}\right]=0 .
\end{aligned}
$$

Now, just as in the case of a single component system, we expand the volume uniformly by $\varepsilon_{\mathrm{V}}$, and seek the condition for local topological instability. Obviously it requires less volume expansion to bring in a smaller $M$ atom than a larger $T$ atom. Thus the critical volume depends on the combination of elements. For instance in order to determine the local volume expansion around a $T$ atom necessary to bring in an $M$ atom to its neighbor, $\varepsilon_{\mathrm{V}}^{T-M}$, we assume $\delta N_{\mathrm{C}}^{T-T}=0$, etc. From (25) and (26),

$$
\begin{gathered}
\delta N_{\mathrm{C}}^{T-T}=-\frac{N_{\mathrm{C}}^{T-T} \delta \omega_{T-T}+N_{\mathrm{C}}^{T-M} \delta \omega_{T-M}}{\omega_{T-T}}=\frac{1}{2} \quad(T \text { neighbor of } T) \\
\delta N_{\mathrm{C}}^{T-M}=-\frac{N_{\mathrm{C}}^{T-T} \delta \omega_{T-T}+N_{\mathrm{C}}^{T-M} \delta \omega_{T-M}}{\omega_{T-M}}=\frac{1}{2} \quad(M \text { neighbor of } T) \\
\delta N_{\mathrm{C}}^{M-T}=-\frac{N_{\mathrm{C}}^{M-T} \delta \omega_{M-T}+N_{\mathrm{C}}^{M-M} \delta \omega_{M-M}}{\omega_{M-T}}=\frac{1}{2} \quad(T \text { neighbor of } M) \\
\delta N_{\mathrm{C}}^{M-M}=-\frac{N_{\mathrm{C}}^{M-T} \delta \omega_{M-T}+N_{\mathrm{C}}^{M-M} \delta \omega_{M-M}}{\omega_{M-M}}=\frac{1}{2} \quad(M \text { neighbor of } M)
\end{gathered}
$$

When the volume is expanded each atomic volume does not change, but gaps in-between increase. In order to calculate the change in the preferred local coordination because of the gaps, we artificially inflate the atom at the center. For instance let us assume that an $M$ atom is at the center and we want to calculate the change in the solid angle to view a $T$ atom due to volume expansion. The change in the $T-M$ distance is $\left(\varepsilon_{\mathrm{V}}^{M-T} / 3\right)\left(r_{T}+r_{M}\right)$. This corresponds to the change in $z$ by $r_{M} d z$, so that $\varepsilon_{\mathrm{V}}^{M-T} / 3=d z /(1+z)$. Similarly,

$$
\begin{aligned}
& \frac{\varepsilon_{\mathrm{V}}^{T-T}}{3}=\frac{d z}{2}, \quad \frac{\varepsilon_{\mathrm{V}}^{T-M}}{3}=-\frac{d z}{z(1+z)}, \\
& \frac{\varepsilon_{\mathrm{V}}^{M-T}}{3}=\frac{d z}{1+z}, \quad \frac{\varepsilon_{\mathrm{V}}^{M-M}}{3}=\frac{d z}{2 z},
\end{aligned}
$$

Thus we obtain,

$$
\begin{aligned}
\delta \omega_{T-T} & =-\frac{\pi \sqrt{3}}{9} \varepsilon_{\mathrm{V}}^{T-T}=-\alpha \varepsilon_{\mathrm{V}}^{T-T}, \\
\delta \omega_{M-M} & =-\frac{\pi \sqrt{3}}{9} \varepsilon_{\mathrm{V}}^{M-M}=-\alpha \varepsilon_{\mathrm{V}}^{M-M}, \\
\delta \omega_{T-M} & =-\frac{2 \pi z^{2}}{3(1+z) \sqrt{1+2 z}} \varepsilon_{\mathrm{V}}^{T-M}=-\beta_{1} \varepsilon_{\mathrm{V}}^{T-M}, \\
\delta \omega_{M-T} & =-\frac{2 \pi}{3(1+z) \sqrt{z(z+2)}} \varepsilon_{\mathrm{V}}^{M-T}=-\beta_{2} \varepsilon_{\mathrm{V}}^{M-T} .
\end{aligned}
$$

We now obtain four critical volume strains,

$$
\begin{aligned}
\varepsilon_{\mathrm{V}}^{T-T} & =\frac{\omega_{T-T}}{2\left[N_{\mathrm{C}}^{T-T} \alpha+N_{\mathrm{C}}^{T-M} \beta_{1}\right]}, \\
\varepsilon_{\mathrm{V}}^{T-M} & =\frac{\omega_{T-M}}{2\left[N_{\mathrm{C}}^{T-T} \alpha+N_{\mathrm{C}}^{T-M} \beta_{1}\right]}, \\
\varepsilon_{\mathrm{V}}^{M-T} & =\frac{\omega_{M-T}}{2\left[N_{\mathrm{C}}^{M-T} \beta_{2}+N_{\mathrm{C}}^{M-M} \alpha\right]}, \\
\varepsilon_{\mathrm{V}}^{M-M} & =\frac{\omega_{M-M}}{2\left[N_{\mathrm{C}}^{M-T} \beta_{2}+N_{\mathrm{C}}^{M-M} \alpha\right]} .
\end{aligned}
$$

They are related by,

$$
\begin{aligned}
& \frac{\varepsilon_{\mathrm{V}}^{T-M}}{\varepsilon_{\mathrm{V}}^{T-T}}=\frac{\omega_{T-M}}{\omega_{T-T}} \approx z, \\
& \frac{\varepsilon_{\mathrm{V}}^{M-M}}{\varepsilon_{\mathrm{V}}^{M-T}}=\frac{\omega_{M-M}}{\omega_{M-T}} \approx z, \\
& \varepsilon_{\mathrm{V}}^{M-M} \approx \varepsilon_{\mathrm{V}}^{T-T} \approx \varepsilon_{\mathrm{V}}^{c r i t} .
\end{aligned}
$$

Thus in terms of the magnitude they are, $\varepsilon_{\mathrm{V}}^{T-M}<\varepsilon_{\mathrm{V}}^{M-M} \approx$ $\varepsilon_{\mathrm{V}}^{T-T}<\varepsilon_{\mathrm{V}}^{M-T}$. In the approximation (40)-(42) we assumed no compositional short-range order (CSRO). As we discuss later this is changed by introducing CSRO. Using these critical strains we can define the compositionally resolved glass transition temperature,

$$
\begin{aligned}
T_{\mathrm{g}}^{T-T} & =\frac{2 \Omega_{T} K_{T}}{k_{\mathrm{B}}}\left(\varepsilon_{\mathrm{V}}^{T-T}\right)^{2}, \\
T_{\mathrm{g}}^{T-M} & =\frac{2 \Omega_{T} K_{T}}{k_{\mathrm{B}}}\left(\varepsilon_{\mathrm{V}}^{T-M}\right)^{2}, \\
T_{\mathrm{g}}^{M-T} & =\frac{2 \Omega_{M} K_{M}}{k_{\mathrm{B}}}\left(\varepsilon_{\mathrm{V}}^{M-T}\right)^{2}, \\
T_{\mathrm{g}}^{M-M} & =\frac{2 \Omega_{M} K_{M}}{k_{\mathrm{B}}}\left(\varepsilon_{\mathrm{V}}^{M-M}\right)^{2},
\end{aligned}
$$

where $\Omega_{T}$ and $K_{T}$ are the local volume and bulk modulus of an element $T$, etc. The local bulk modulus, $K$, depends upon the interatomic potential. ${ }^{22}$ Thus the local glass transition temperature depends upon the local interaction through $K$ (chemical effect) and the atomic size through $\Omega$ and $\varepsilon_{\mathrm{V}}$ (size effect).

\section{Glass Transition and Fragility of a Liquid}

\subsection{Viscosity of a liquid}

We are now ready to discuss the viscosity of a liquid as it relates to glass transition and fragility. Let us consider what the compositionally resolved glass transition temper- 
ature physically means. It is the temperature above which the local volume fluctuation is large enough to allow another atom to come into the nearest neighbor shell. For the sake of simplicity let us assume the product $\Omega K$ is the same for $T$ and $M$. Then we find, $T_{\mathrm{g}}^{T-M}<T_{\mathrm{g}}^{M-M} \approx T_{\mathrm{g}}^{T-T}<T_{\mathrm{g}}^{M-T}$. As the liquid alloy is cooled, at first the $T$ neighbors of $M$ become frozen, i.e. the $T-M$ bond has a life time longer than a period of phonon (Debye) oscillation. Then the $M$ neighbors of $M$ and $T$ neighbors of $T$ become frozen, and finally $M$ neighbors of $T$ becomes frozen, and the whole system becomes glassy. Thus glass transition in a multi-component system does not occur uniformly, but gradually over a range of temperature reflecting the inhomogeneity at the atomic level. This dispersion of the glass transition is an important key in determining the viscosity and fragility of a liquid. If the compositionally resolved glass transition temperatures are widely dispersed the liquid will gradually freeze over a wide range of temperature, and thus will exhibit a strong liquid behavior. On the other hand if the dispersion is small it will be a fragile liquid. Thus alloy selection should aim at increasing the range of glass transition temperatures.

\subsection{Local glass transition and the nano-glass state}

Let us now examine the four glass transition temperatures a little more carefully. Inspecting eqs. (36)-(46) it is clear that the glass transition temperature to bring in $M$ atoms, which are smaller in size, is always lower than that for $T$ atoms. This is because the volume expansion to bring in a smaller $M$ atom is always less than that for a larger $T$ atom. This means that $M$ atoms have larger mobility than $T$ atoms, which is intuitively obvious because smaller $M$ atoms diffuse faster via the interstitial mechanism. Then the local glass transition at $T_{\mathrm{g}}^{M-T}$ is irrelevant, because the local environment of $M$ atoms will not freeze until the temperature is lowered to $T_{\mathrm{g}}^{M-M}$. Thus the $l o$ cal glass transition around a $M$ atom occurs at $T_{\mathrm{g}}^{M}=T_{\mathrm{g}}^{M-M}$. The local glass transition temperature of a $T$ atom depends upon the composition. When the chemical composition of $M$ atoms is small, the mobility of a $M$ atoms will not affect the dynamics of the majority element $T$, so that $T_{\mathrm{g}}^{T} \approx T_{\mathrm{g}}^{T-T}$. Thus,

$$
\frac{T_{\mathrm{g}}^{M}}{T_{\mathrm{g}}^{T}}=\frac{T_{\mathrm{g}}^{M-M}}{T_{\mathrm{g}}^{T-T}}=\frac{\Omega_{M} K_{M}}{\Omega_{T} K_{T}}\left(\frac{\varepsilon_{\mathrm{V}}^{M-M}}{\varepsilon_{\mathrm{V}}^{T-T}}\right)^{2} \approx \frac{\Omega_{M} K_{M}}{\Omega_{T} K_{T}} .
$$

If $\Omega K$ of $T$ and $M$ are similar, $T_{\mathrm{g}}^{M} \approx T_{\mathrm{g}}^{T}$, and the system will have a single glass transition given by,

$$
T_{\mathrm{g}} \approx T_{\mathrm{g}}^{M-M} \approx T_{\mathrm{g}}^{T-T} \approx \frac{2 \Omega K}{k_{\mathrm{B}}}\left(\varepsilon_{\mathrm{V}}^{\text {crit }}\right)^{2}=6.14 \times 10^{-3} \frac{\Omega K}{k_{\mathrm{B}}},
$$

just as in a single component glass, and the liquid will show a fragile behavior, with the viscosity changing rapidly with temperature. Now note that $\Omega K$ provides the temperature scale for structural fluctuation as in (17), therefore should scale with the melting temperature $T_{M}$ as long as the anharmonicity is similar. Thus choosing components with greatly different melting temperature should create the gap between $T_{\mathrm{g}}^{M}$ and $T_{\mathrm{g}}^{T}$, and results in the strong liquid behavior. Since the glass transition of the whole system will nearly coincide with that of the majority component, $T$, choosing a metalloid with a high melting temperature, such as boron and silicon, is an important recipe.

Changing the $\Omega K$ product, which depends upon the atomic interaction, is a chemical approach. It is also possible to utilize the atomic size effect by manipulating the CSRO. For instance if we introduce repulsion between $M$ atoms and eliminate $M-M$ pairs $\left(N_{\mathrm{C}}^{M-M}=0\right)$, the environment of $M$ will freeze at $T_{\mathrm{g}}^{M}=T_{\mathrm{g}}^{M-T}$ rather than $T_{\mathrm{g}}^{M-M}$. This creates a gap between the freezing temperatures of $T$ and $M$ atoms even when $\Omega K$ is similar. From eqs. (40)-(46),

$$
\frac{T_{\mathrm{g}}^{M}}{T_{\mathrm{g}}^{T}}=\frac{T_{\mathrm{g}}^{M-T}}{T_{\mathrm{g}}^{T-T}}=\frac{\Omega_{M} K_{M}}{\Omega_{T} K_{T}}\left(\frac{\varepsilon_{\mathrm{V}}^{M-T}}{\varepsilon_{\mathrm{V}}^{T-T}}\right)^{2} \approx \frac{\Omega_{M} K_{M}}{\Omega_{T} K_{T}} \frac{1}{z^{2}} .
$$

This ratio is plotted in Fig. 4 for $\mathrm{T}_{0.8} \mathrm{M}_{0.2}$ alloy as a function of $z$, along with the approximations, $1 / z^{2}$ as well as $1 / z^{1.7}$. Also $N_{\mathrm{C}}^{T-T}, N_{\mathrm{C}}^{T-M}$ and $N_{\mathrm{C}}^{M-T}$ are shown in Fig. 5, and $\varepsilon_{\mathrm{V}}^{T-T}$ and $\varepsilon_{\mathrm{V}}^{M-T}$ in Fig. 6. In this calculation we assumed that $\Omega_{T} K_{T}=\Omega_{M} K_{M}$, and $N_{\mathrm{C}}^{M-M}=0$. Note that in (49) the chemical effect due to the ratio of $\Omega K$ is compounded with the size effect described by the ratio of $\varepsilon_{\mathrm{V}}$. In this picture the environment of $M$ freezes above $T_{\mathrm{g}}^{T}$, so that each $M$ atom forms a local cluster of $M T_{n}$, with $M$ at the center. This cluster is not a chemical molecule that results from chemical bonds. It is simply a product of local freezing, a nano-glass. The liquid between $T_{\mathrm{g}}^{T}$ and $T_{\mathrm{g}}^{M}$ is a composite of nano-glasses and liquid, and should exhibit a high viscosity if the concentration of the nano-glass is above the percolation concentration. This would result in a strong liquid behavior, and the system should be easily cast into a bulk metallic glass. Thus the key in producing a strong liquid is to have a large ratio of the local glass transition temperatures, eq. (49).

In summary, the gap between the two local glass transition temperatures can be widened by the following:

1. Decrease the magnitude of $z$.

2. Increase the local bulk modulus of $M$ in the environment of $T$ by increasing the strength of interaction between $T$ and $M$.

3. Introduce repulsive interaction between small atoms.

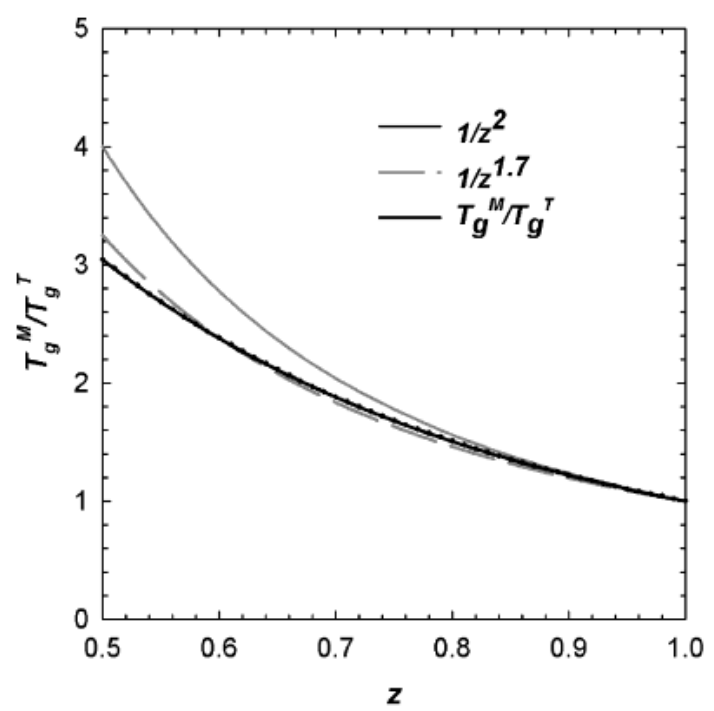

Fig. 4 Ratio of the local glass transition temperatures, $T_{\mathrm{g}}(M) / T_{\mathrm{g}}(T)$, eq. (47), calculated for $\mathrm{T}_{0.8} \mathrm{M}_{0.2}$ alloy by assuming strong $M-M$ repulsion. 


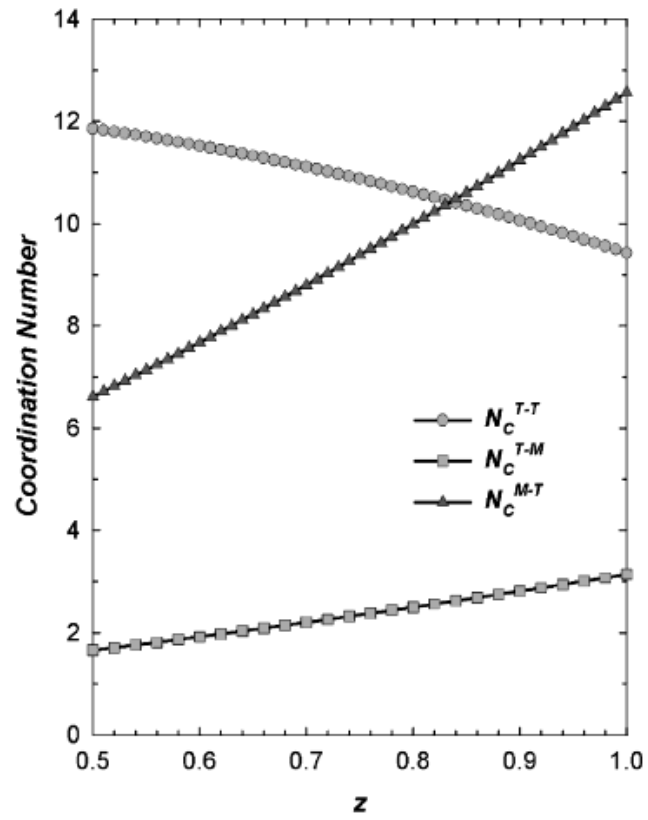

Fig. 5 Compositionally resolved local coordination numbers for the same system.

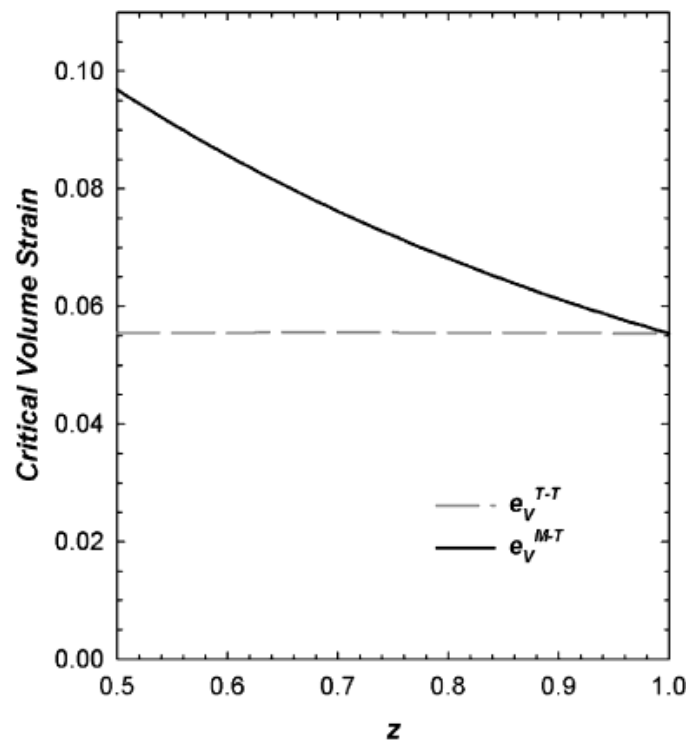

Fig. 6 Critical volume strain for $T$ and $M$ atoms. Note that $\varepsilon_{\mathrm{V}}^{T-T}$ is nearly independent of $z$, as suggested by eq. (42), but $\varepsilon_{\mathrm{V}}^{M-T}$ increases with decreasing $z$, as predicted by (41).

Actually alloys of transition-metal and metalloid, such as boron and phosphorus, satisfy these conditions. There is a strong interaction between $T$ and $M$ involving charge transfer, or the hybridization of the $T d$-orbitals and the $M s-p$ orbitals, resulting in the shortened bond between $T$ and $M$ and repulsion between $M .^{28,30)}$ Glass formability is further improved by adding more elements with larger size differences, such as $\mathrm{Zr}$ and $\mathrm{Nb}$ into $\mathrm{Fe}-\mathrm{B}{ }^{31}$ )

The idea of local glass transition is strongly supported by recent results on the NMR measurement of diffusivity in complex liquid by Tang et al. ${ }^{32)}$ They found that in $(\mathrm{Zr}, \mathrm{Ti})(\mathrm{Cu}, \mathrm{Ni}) \mathrm{Be}$ bulk metallic glasses the jump frequency of Be atom did not show any anomaly in temperature dependence through $T_{\mathrm{g}}$, even though the diffusivity shows a sharp change at $T_{\mathrm{g}}$. As far as local jumps of $\mathrm{Be}$ is concerned the liquid above $T_{\mathrm{g}}$ appears still glassy. This is a direct proof that the environment of $\mathrm{Be}$ is frozen even above $T_{\mathrm{g}}$, so that a $\mathrm{Be}$ atom moves only collectively, accompanied by the motion of a nearest neighbor cluster.

\subsection{Glass formability}

The problem of the ease of glass formation has two facets, the kinetic aspect and the energetic aspect. The concept of the distributed local glass transitions addresses the kinetic aspect, while the energetic aspect is addressed by the concept of local atomic level strains proposed earlier. ${ }^{8)}$ The driving force for crystallization is reduced by increasing the atomic level elastic strain energy of a crystal. This can be achieved by the following:

1. Decrease the magnitude of $z$.

2. Increase the number of elements involved.

These prescriptions are supported by the fact that as many as 5 or 6 elements with a large variation in sizes are involved in the bulk metallic glass composition. They also overlap with the requirements for improving the kinetics as discussed above.

\section{Conclusions}

The theory of glass transition based upon the concept of local topological instability of the atomic structure was extended to deal with multi-component glasses. It was shown that a multi-component liquid freezes gradually over a range of temperature, reflecting the atomic level compositional inhomogeneity. A wide distribution of the local glass transition temperatures results in more gradual freezing and formation of a nano-glass state, and thus the strong-glass behavior as defined by Angel. In particular, a repulsive potential between small $M$ atoms and the attraction between a small $M$ and large $T$ atoms result in the local glass transition temperature of $M$ atoms much higher than the global glass transition temperature. This creates atomic clusters around $M$ atoms in the liquid (nano-glasses), and increases the viscosity of the liquid. By considering both the kinetic and energetic aspects of glass formation, four conditions were identified as the principles to facilitate bulk metallic glass formation:

1. Increase the atomic size ratio of the constituent elements.

2. Increase the number of elements involved.

3. Increase the interaction between the small and large atoms.

4. Introduce repulsive interaction between small atoms.

Not surprisingly, these principles are already in use in the effort to identify the metallic glass alloy compositions, except that the importance of the $M-M$ repulsion may not have been recognized so far. The present theory validates these principles and places them on a firmer ground.

\section{Acknowledgments}

The author has greatly benefited from discussions with W. L. Johnson and J. S. Poon. This work was supported by the Defense Advanced Research Project Agency and the Office Naval Research through DARPA/ONR Grant N00014-01-10961 . 


\section{REFERENCES}

1) J. Jäcle: Rep. Prog. Phys. 49 (1986) 171-231.

2) W. Götze and L. Sjögren: Rep. Prog. Phys. 55 (1992) 241-376.

3) M. H. Cohen and D. Turnbull: J. Chem. Phys. 31 (1959) 1164-1169.

4) D. Turnbull and M. H. Cohen: J. Chem. Phys. 34 (1961) 120-125.

5) D. Turnbull and M. H. Cohen: J. Chem. Phys. 52 (1970) 3038-3041

6) A. I. Taub and F. Spaepen: Acta Metall. 28 (1980) 1781-1788.

7) A. L. Greer and F. Spaepen: Ann. N. Y. Acad. Sci. 371 (1981) 218-237.

8) T. Egami and Y. Waseda: J. Non-Crsyt. Solids 64 (1984)113-134.

9) T. Egami: Mater. Sci. Eng. A226-228 (1997) 261-267.

10) C. A. Angel: Science 267 (1995) 1924-1935.

11) A. Inoue, T. Zhang and T. Masumoto: Mater. Trans., JIM 31 (1990) 425-428.

12) T. Zhang, A. Inoue and T. Masumoto: Mater. Trans., JIM 32 (1991) 1005-1010

13) A. Peker and W. L. Johnson: Appl. Phys. Lett. 63 (1993) 2342-2344.

14) R. Busch, A. Masuhr, E. Bakke and W. L. Johnson: Mater. Sci. Forum 269-272 (1998) 547-552.

15) W. H. Zachariasen: J. Am. Ceram. Soc. 54 (1932) 3841-3845.

16) Y. Suzuki and T. Egami: J. Non-Cryst. Solids 75 (1985) 361-366.

17) Y. Suzuki, J. Haimovich and T. Egami: Phys. Rev. B 35 (1987) 2162-
2168.

18) T. Egami and S. Aur: J. Non-Cryst. Solids 89 (1987) 60-74.

19) J. L. Tallon: Nature (London) 299 (1982) 188-188.

20) F. A. Lindemann: Phys. Z. 11 (1910) 609-615.

21) T. Egami, K. Maeda and V. Vitek: Philos. Mag. A 41 (1980) 883-901.

22) T. Egami and D. Srolovitz: J. Phys. F, 12 (1982) 2141-2163; J. Phys. F, 13 (1983) 909 (Corrigendum).

23) S.-P. Chen, T. Egami and V. Vitek: Phys. Rev. B 37 (1987) 2440-2449.

24) M. H. Cohen and G. S. Grest: Phys. Rev. B 20 (1979) 1077-1098.

25) G. S. Grest and M. H. Cohen: Phys. Rev. B 21 (1980) 4113-4117.

26) T. Egami, K. Maeda, D. Srolovitz and V. Vitek: J. Phys. (Paris) 41 C8 (1980) 272-275.

27) R. Zallen: The Physics of Amorphous Solids, (Wiley, New York, 1983).

28) T. Egami: Rep. Prog. Phys. 47 (1984) 1601-1725.

29) G. S. Cargill and F. Spaepen: J. Non-Cryst. Solids 43 (1981) 91-97.

30) R. A. Alben, J. I. Budnick and G. S. Cargill, III: in Metallic Glasses, ed. J. J. Gilman and H. J. Leamy (Metals Park, OH, 1978) p. 304-334.

31) A. Inoue, K. Kobayashi, J. Kanehira and T. Masumoto: Sci. Rep. Res. Inst. Tohoku Univ. 29A (1981) 331-342.

32) X. P. Tang, U. Geyer, R. Busch, W. L. Johnson and Y. Wu: Nature (London) 402 (1999) 160-162. 